\title{
Catastrophic failure of polymer melts during extension
}

\section{Rasmussen, Henrik K.}

\section{Published in:}

Journal of Non-Newtonian Fluid Mechanics

Link to article, DOI:

10.1016/j.jnnfm.2013.05.001

Publication date:

2013

Link back to DTU Orbit

Citation (APA):

Rasmussen, H. K. (2013). Catastrophic failure of polymer melts during extension. Journal of Non-Newtonian Fluid Mechanics, 198, 136-140. https://doi.org/10.1016/j.jnnfm.2013.05.001

\section{General rights}

Copyright and moral rights for the publications made accessible in the public portal are retained by the authors and/or other copyright owners and it is a condition of accessing publications that users recognise and abide by the legal requirements associated with these rights.

- Users may download and print one copy of any publication from the public portal for the purpose of private study or research.

- You may not further distribute the material or use it for any profit-making activity or commercial gain

- You may freely distribute the URL identifying the publication in the public portal

If you believe that this document breaches copyright please contact us providing details, and we will remove access to the work immediately and investigate your claim 


\title{
Catastrophic Failure of Polymer Melts During Extension
}

\author{
Henrik Koblitz Rasmussen \\ Department of Mechanical Engineering \\ Technical University of Denmark. DK-2800 Kgs. Lyngby, Denmark
}

\begin{abstract}
Numerical flow modeling has been applied to study the break of monodisperse polymer melts during extension. These continuum mechanical based computations are within the ideas of the microstructural 'interchain pressure' theory. Calculated breaks, a result of small initial sample imperfections, agree with experimental observations.
\end{abstract}

\section{Introduction}

It is well known that rupture phenomena exist in polymer melts. They appear as failures in extended polymer melts $[1,2,3]$, development of holes in thin films [4, 5], sharkskin etc.. Most of these observations are unresolved and they do not occur in common liquids [5, 6]. The ruptures have been observed to be of either brittle or a flowing nature. The latter one is commonly referred to as a Considere type of failure in extension, where a necking occurs on the extended sample, eventually evolving into a break. The brittle type of failure is experimentally observed as a failure evolving faster than the sampling rate of the equipment [1].

The first effort to obtain an understanding of rupture in polymer liquids and melts dates back to Ziabicki and Takserman-Krozer in 1964 [7] and Chang and Lodge in 1971 [8]. The quoted papers in the section above, and this paper as well, all refer to experimental investigations based on monodisperse polymer melts. These studies on a theoretical ideal material were initiated by Vinogradov and coworkers in the seventies [1].

The first effort to obtain an exact understanding of failures in monodisperse polymer melts, explaining them as of cohesive nature, was published by Joshi and Denn in 2003 [9]. Currently it seems to be the only existing quantitative theory for a cohesive type of failure in polymer melts, although other failure mechanisms have been suggested [2] more recently. These studies indicate the need for an additional explanation for the rupture mechanism upon the fluid dynamics of polymer melts, where the flow should depend solely on the non-equilibrium configurations of the molecules. Actually the theory by Joshi and Denn $[9,10]$ where challenged the same year as it appeared by Bach et al. [11]. They extended monodisperse polystyrenes experimentally, using the filament stretch rheometer [12] without observing any breaks, where the theory by Joshi and Denn [9, 10] predicts the existence of cohesive failures during this extension. To establish a quantitative explanation of the failure during extension I will follow the ideas of Rasmussen and Yu [6]. They successfully predicted the delayed failure (or the lack of it) of a cylinder of fixed length, consisting of a pre-extended monodisperse polymer melt.

There have been remarkable advances in the understanding of the fluid dynamics of polymer melts in the recent years $[13,14,15,16,17,18]$, as new experimental techniques have appeared [12, 19]. 
The purpose of this study is twofold. First of all, to study whether the failure in extension can be explained within the recent theoretical insight in the dynamics of polymers, in order to obtain a quantitative agreement with experimental observations. Secondarily to discuss its consequences for the suggested cohesive type of failure mechanisms. As in most experimental studies $[1,2,3,11,20,21]$ the focus will be on the extension and potential breakup of monodisperse polymer melts, applying narrow molecular weight distributed polystyrene (PS), polyisoprene (PI) and styrenebutadiene (SBR) melts.

\section{Polymer melt dynamics}

The recent constitutive equation by Wagner et al. [14, 22] based on the 'interchain pressure' concept [13] is applied here. This approach accurately predicts the startup and steady shear [22] as well as extensional flow [14], potentially followed by stress relaxation [23,6], of monodisperse polymer melts. This has currently been documented up to about 160 entanglements in shear [22] and at least 30 in extensional flow [14]. It has the ability to predict large strain reversed [24] including oscillatory [25] flow as well. Although an immense variety of constitutive concepts have been published in the past [26, 27, 28, 29], only models based on the 'interchain pressure' concept are capable of predicting this dynamic of monodisperse melts $[14,16]$. Note that the number of entanglements are defined as $Z=M_{w} / M_{e}$, where $M_{w}$ and $M_{e}$ are the weight average and the entanglement molecular weight, respectively. The components of the stress tensor $\left(\sigma_{\mathrm{ij}}\right)$ are given as

$$
\begin{gathered}
\sigma_{i j}=\int_{-\infty}^{t} M\left(t-t^{\prime}\right) f\left(t, t^{\prime}\right)^{2} 5\left\langle\frac{E_{i n} u_{n} E_{j m} u_{m}}{|\mathbf{E} \cdot \mathbf{u}|^{2}}\right\rangle d t^{\prime}, \\
\frac{\partial}{\partial t} f\left(t, t^{\prime}\right)=f\left(t, t^{\prime}\right)\left[\frac{\partial}{\partial t}\langle\ln |\mathbf{E} \cdot \mathbf{u}|\rangle-\frac{f\left(t, t^{\prime}\right)}{3 \tau_{R}}\left(f\left(t, t^{\prime}\right)^{3}-1\right)\right]
\end{gathered}
$$

and $f\left(t^{\prime}, t^{\prime}\right)=1$. $f$ is referred to as the molecular stress function. The angular brackets denote an average over a unit sphere $\langle\ldots\rangle=1 /(4 \pi) \int_{|\mathbf{u}|=1} \ldots d \mathbf{u} . \mathbf{u}$ is a unit vector. The components of the displacement gradient tensor, $\mathbf{E}$, are given by $E_{\mathrm{ij}}\left(\boldsymbol{x}, t, t^{\prime}\right)=\partial x_{\mathrm{i}} / \partial x_{\mathrm{j}}{ }^{\prime}, i=1,2,3$ and $j=1,2,3$. $\left(x_{1}^{\prime}, x_{2}^{\prime}, x_{3}^{\prime}\right)$ are the Cartesian coordinates of a given particle in the stress free reference state (time $\left.t^{\prime}\right)$, displaced to coordinates $\left(x_{1}, x_{2}, x_{3}\right)$ in the current state (time $t$ ). $\tau_{R}$ is the Rouse time and $M\left(t-t^{\prime}\right)$ the memory function containing the linear dynamics of the polymer. The Rouse time uniquely defines the nonlinear dynamic of the melt, although the actual value may depend somewhat on the used definition. The used definition is based on experimental evidence, as discussed in the following section 4 .

The memory function by Baumgaertel, Schausberger and Winter (BSW) [30] is used to describe the linear dynamics. Omitting the glassy part, the memory function

$$
M\left(t-t^{\prime}\right)=\int_{0}^{\lambda_{\max }} n_{e} G_{N}^{0}\left(\frac{\lambda}{\lambda_{\max }}\right)^{n_{e}} \frac{e^{\left(t-t^{\prime}\right) / \lambda}}{\lambda^{2}} d \lambda .
$$

$\lambda_{\max }$ is the maximal relaxation time in an elsewhere continuous distribution and $G_{N}^{0}$ the plateau modulus. $n_{e}$ has a unique value for each type of polymer. The BSW model is the currently most accurate method to fit small amplitude oscillatory data.

\section{Modeling}

The published breakup studies were performed with a variety of extensional equipment. Basically, these were either the extension of a cylindrical shaped sample from the ends of the sample $[1,11]$ 
with a fixture moving in the axial (e.g. extensional) direction, or the use of a dual-wind up system $[2,3,20,21]$. Both cylindrical $[2,20]$ and rectangular strips [3] were applied in the dual-wind system. In these systems, the extension was performed by rolling samples upon two (fixed) counter rotating systems, rotating with the same rate. In any extension, it is important to distinguish between the real strain $(\epsilon)$ and the set strain $\left(\epsilon_{N}\right)$ on the equipment. The real kinematic strain is defined as $\epsilon(t)=\ln (l(t) / l(0))$, where $l(t)$ and $l(t=0)$ are the axial (e.g. $z$ ) distances between two narrow particles in the direction of the extension. Assuming constant extension rates $\epsilon=\dot{\epsilon} \cdot t$ or $\epsilon_{N}=\dot{\epsilon}_{N} \cdot t$.

The complexity of the contact condition toward the extension fixtures is avoided in the numerical modeling. The initial shape of the specimen is a cylinder with initial radius $R$ and length $L(t=0)$. Symmetry is applied at the ends of the samples $(z= \pm L / 2)$ and at the center $(z=0)$. The axial coordinates of the ends of the cylinder $(z= \pm L / 2)$ are extended exponentially in time to achieve the extension. Axis symmetry is assumed in applying axial $(z)$ and radial $(r)$ coordinates. The boundary conditions on the free surfaces are no exertion of stress and pressure, and a neglectable surface tension. The sample is stress free and at rest initially (at $t=0$ ).

A necking on a sample would be considered an evolution of an instability in a traditional continuums mechanical framework. A classical linear instability analysis has the ability to predict if a break does not appear due to its validity in the range of small deviation from the ideal case [31]. Unfortunately a classical instability analysis is incapable of predicting the nonlinear evolution of a necking and particularly its potential breakup. For polymer melts and entangled solutions it may be more precise to refer to a sensitivity to the initial conditions. Non ideal conditions will develop in the preparation and handling of the polymer. Here the initial deviation from an ideal cylinder is imposed on the center of the sample $(z=0)$, although an observed break has not been reported to appear at any specific place on the sample [1, 2, 32]. Some theoretical studies suggest that the necking should be positioned at the center of the sample on theoretical ideal geometries [33, 34]. The deviation from the ideal cylinder in the initial geometry is a sinusoidal shaped suppression in the surface with a depth of $H$ and a width of $2 W$ in the axial direction as illustrated in figure 1 .

Computationally, the sample dynamics is evaluated relatively to the sample radius as the effect of the surface tension $(\sigma)$ is neglected. Using the surface elastic number, the elasticity relative to the surface tension [35], a characteristic curvature of less than $\sigma / G_{N}^{0} \approx 0.2 \mu \mathrm{m}$ is obtained. Therefore the absolute size of the initial deviation becomes important and will affect the dynamic of the necking, as its curvature approaches a few micrometer.

The numerical modeling of the extension of a sample with axially moving fixture is straightforward. The length of the sample is specified as $L(t)=L_{0} \exp (\epsilon)$, where $L(t=0)=L_{0}$ is the initial length of the actual extended cylinder. It is more cumbersome to define the initial sample length $(L(t=0))$ in a dual-wind up computation. In a dual-wind up system the unsupported (e.g. extended) part of the sample is ideally a fixed value, here defined as $L_{0}$. The length of the sample in the computations should ideally be $L_{0}$ at the point where the sample break. By definition a break is where the cross sectional area goes to zero in finite time and the strain to obtain a (potential) break is $\epsilon_{c}$. The cylinder is therefore extended as $L(t)=L_{0} \exp \left(\epsilon-\epsilon_{c}\right)$. In this definition the initial length $L(t=0)=L_{0} \exp \left(-\epsilon_{c}\right)$ is implicitly given, as the strain of the break $\epsilon_{c}$ is unknown prior to the start of the extension. An iteration procedure is therefore applied to obtain the corresponding $\epsilon_{c}$ and $L(t=0)$ values, if the solution exists.

In the numerical modeling, the finite element method from Rasmussen [36, 37] is used, solving the momentum balance and volume conservation equations simultaneously. It utilizes a Lagrangian kinematic, e.g. a particle description, as in the applied constitutive equation (1) and (2). Notice that the Currie approximation [38] is applied to all the terms represented by the angular brackets in equation (1) and (2) in all the finite element computations. A computation of the dynamics of the surface during an extension is shown in figure 1. A clear necking phase occurs, evolving into a break.

Very few papers $[2,20,32]$ have reported the appearance of the sample dynamics leading to the 
break in monodisperse melts. In these cases the surface development are of a similar character, although the real appearances represent more complicated surface shapes. In the computations the initial sinusoidal shaped suppression represents a simple deviation from an ideal surface, and it continues to evolve as geometrically simple suppression in the surface.

\section{Breakup}

A break appears when the cross sectional area goes towards zero. Computationally, it is not possible to find this limit exactly by using a finite element method (see figure 2). To define a break computationally the same definition as in Rasmussen and $\mathrm{Yu}$ is applied: A break occurs if a linear extrapolation of the (smallest) cross sectional area of the sample, $A$, versus the time, $t$, reaches zero for a sufficiently small change in time. This may be written as the Hencky strain $\epsilon_{c}=\epsilon$ where $A \cdot d \epsilon / d A<\delta \cdot \epsilon$ and $\delta$ is sufficiently small. Here a $\delta$ value of 0.01 is used, e.g. the actual break is expected to occur less than 1\% later in Hencky strain units. This represents an insignificant difference in between the theoretically real break and the calculated break.

The currently most extensive breakup study was performed by Wang and co-workers [20] on a $95 \mathrm{~kg} /$ mole SBR melt, using a dual-wind up system of the SER type where $L_{0}=12.7 \mathrm{~mm}$. The critical strain of break $\epsilon_{c}$ is shown in figure 3, for a $R=1 \mathrm{~mm}$ cylindrical shaped sample. Cylindrical samples with an initial radius of $R=1 \mathrm{~mm}$ need a correction of $\epsilon=0.9 \cdot \epsilon_{N}$ [39], resulting in relation of $\dot{\epsilon}=0.9 \cdot \dot{\epsilon}_{N}$ for the corresponding rates. In figure 3 the strain of break is shown as a function of the elongational rate multiplied by the maximal relaxation time. The corresponding computations of $\epsilon_{c}$ with the material parameters obtained by Rasmussen and $\mathrm{Yu}$ [6] and Lyhne et al. [40] (table 1) are shown in figure 3 as well. I apply the same initial deviations from an ideal cylinder $(H / R=0.015,0.05$ and $W / R=0.2)$ as in Rasmussen and $\mathrm{Yu}[6]$, where they explained the spontaneous rupture in polymer melts. As in Rasmussen and $\mathrm{Yu}$ [6] only the sample height has been changed, as the computations are considerably less sensitive to changes in the width. In experiments, imperfections on the samples will not be exactly the same, although the break is not very sensitive to the size of the disturbance. The computations show agreement with the observations on the whole range of flow rates. They predict the steep descent in the stability near the Newtonian flow area at low strain rates $\left(\lambda_{\max } \cdot \dot{\epsilon}<1\right)$. The break occurs at very low strain values in the area $\left(1<\lambda_{\max } \cdot \dot{\epsilon}<\lambda_{\max } / \tau_{R} \approx 78\right)$ dominated by configurational stress. Finally, the necking is stabilized in the strain hardening regime $\left(78 \approx \lambda_{\max } / \tau_{R}<\lambda_{\max } \cdot \dot{\epsilon}\right)$.

Contrary to the study on the $95 \mathrm{~kg} /$ mole SBR melt by Wang and Wang [20], Bach et al. (2003b) [11] extended a $390 \mathrm{~kg} /$ mole PS up to strains in between 3.6 and 5 without observing any break. Wang and Wang [20] and Bach et al. [11] measured at about the same $\lambda_{\max } \cdot \dot{\epsilon}$ values, and the 95 $\mathrm{kg} /$ mole SBR melt has almost identical flow behavior as a $390 \mathrm{~kg} / \mathrm{mole}$ PS [6], of course relative to $\lambda_{\max }$. Bach et al. [11] extended a short cylindrical sample. They measured and feedback controlled the local extension exactly at the neck of the sample, ensuring a pre-described extension at the neck. This avoids a break, contrary to all the other published studies where the overall sample was extended exponentially in time. An almost similar PS (of $465 \mathrm{~kg} / \mathrm{mole}$ ) extended by Luap et al. [3] failed catastrophically. They extended rectangular shaped strips using a dual wind rheometer (of the RME type). The measurement range was limited and the actual break appears at slightly higher strain values than the reported points in figure 3 . The $390 \mathrm{~kg} / \mathrm{mole}$ PS [6] was reported to have $Z=29$ entanglements and the $465 \mathrm{~kg} /$ mole PS $Z=34$ entanglements. Applying the well-established relation $\lambda_{\max } \propto Z^{3.4}$ from Milner and McLeish [41] the time constants in table 1 actually indicates a difference of 3 entanglements between the melts. In most definitions the Rouse time follows a relation of $\tau_{R} / \lambda_{\max } \propto 1 / Z$. Therefore these 3 entanglements represent an insignificant difference, as observed experimentally [3]. The currently suggested breaking mechanisms of cohesive nature $[2,9]$ 
are unable to predict the above behavior. The break in the PS would therefore be expected to be of a fluid dynamic nature.

The effect of an increased number of entanglements has been illustrated in figure 3 . The only non-dimensional parameter of importance is the Rouse time, of course relative to relaxation time. It depends solely on the number of entanglements. The $95 \mathrm{~kg} /$ mole SBR melt studied in Wang and Wang [20] was reported [6] to have $Z=24$ entanglements, whereas a SBR melt with $Z=98$ entanglements (corresponding to the $240 \mathrm{~kg} / \mathrm{mole}$ SBR melt from Wang et al. [21]) is expected to have a Rouse time of $\tau_{R}=(24 / 98) \cdot(1.34 s / 57.95 s) \lambda_{\max } \approx 0.48 \mathrm{~s}$. The increase in entanglement number has destabilizing effect at all extensional rates. Actually, very little experimental evidence exist of the startup and particularly the steady extensional flow behavior of monodisperse polymer melts above $Z=34$ entanglements [6]. For the sake of completeness figure 4 show the theoretically expected and measured extensional viscosities for the $240 \mathrm{~kg} /$ mole SBR melt from Wang et al. (2011) [21], showing the expected agreement.

Figure 5 contains the break of rectangular shaped $240 \mathrm{~kg} /$ mole SBR samples from Wang et al. [21]. These are shown together with the first published breaking study by Vinogradov et al. [1] (data from their figure 1). They extended 'dog-bone' shaped PI, with a central cylinder of length $L_{0}=30 \mathrm{~mm}$ and a radius of $R=2.5 \mathrm{~mm}$, from the ends of the sample. Due to the large aspect ratio it is assumed that the set strain is identical to the real one (e.g. $\epsilon=\epsilon_{N}$ ). The original paper from Vinogradov et al. in 1972 [42] reports a $M_{w}=575$ expected to have $Z=119$ [43], although the time constant in table 1 indicates a $Z=105$ [43]. A similar relative rouse time in the PI melt and the SBR melt with $Z=98$ entanglements would therefore be expected. The calculated breaks for the PI melt are shown in figure 5. The imposed disturbances correspond (non-dimensionally) to the ones in figure 3. The SBR data show a large scattering, whereas the data from Vinogradov et al. [1] show agreement with the calculated break, within the scattering of the measurements.

Of course information of the actual initial conditions of the sample is needed in order to get a computational agreement with each of the individually observed breaks. This allows modeling of the surface dynamics within the experimental accuracy as shown in Bach et al. [44].

Further the intrinsic nature of the applied constitutive equation is not expected to be unique. It is able to predict the dynamics of virtually all published rheometric data for monodisperse melts, but other models are most likely capable of showing a similar agreement and a similar dynamic behavior.

\section{Conclusion}

The breakup of cylindrical shaped monodisperse polymer melts during extension was modeled, evaluating the published breakup studies. To summarize, the break (and the lack of it) of monodiserse polymer melts can be explained within the framework of its fluid mechanics. This is currently the only quantitative theory able to predict the break of (monodisperse) polymers, and there seems to be no need for any additional physical explanation.

\section{References}

[1] G. V. Vinogradov, A. Y. Malkin, and V. V. Volosevitch, Flow, high-elastic (recoverable) deformation, and rupture of uncured high molecular weight linear polymers in uniaxial extension, J. Polymer Sci. 13 1721-1735 (1975).

[2] Y. Wang, P. Boukany, S.-Q. Wang, and X. Wang, Elastic breakup in uniaxial extension of entangled polymer melts, Phys. Rev. Lett. 99, 237801 (2007). 
[3] C. Luap, C. Müller, T. Schweizer, and D. C. Venerus, Simultaneous stress and birefringence measurements during uniaxial elongation of polystyrene melts with narrow molecular weight distribution, Rheol. Acta 45, 83-91 (2005).

[4] J. Becker, G. Grün, R. Seemann, H. Mantz, K. Jacobs, K. R. Mecke, and R. Blossey, Complex dewetting scenarios captured by thin-film models, Nature Materials 2, 59-62 (2003).

[5] K. Yu, H. K. Rasmussen, J. M. R. Marin, and O. Hassager, Mechanism of spontaneous hole formation in thin polymeric films, Phys. Rev. B 85, 024201 (2012).

[6] H. K. Rasmussen, and K. Yu, Spontaneous breakup of extended monodisperse polymer melts, Phys. Rev. Lett. 107, 126001 (2011).

[7] A. Ziabicki, and R. Takserman-Krozer, Mechanism of breakage of liquid threads , KolloidZeitschrift und Zeitschrift für Polymere, 198, 60-65, 1964

[8] H. Chang, A. S. Lodge, A possible mechanism for stabilizing elongational flow in certain polymeric liquids at constant temperature and composition, Rheol. Acta, 10, 448-449 (1971).

[9] Y. M. Joshi and M. M. Denn, Rupture of entangled polymeric liquids in elongational flow, J. Rheol. 47, 291-298 (2003).

[10] Y. M. Joshi and M. M. Denn, Rupture of entangled polymeric liquids in elongational flow with dissipation, J. Rheol. 48, 591-598 (2004).

[11] Bach, A., K. Almdal, H. K. Rasmussen, and O. Hassager, Elongational viscosity of narrow molar mass distribution polystyrene, Macromolecules 36, 5174-5179 (2003b).

[12] A. Bach, H. K. Rasmussen, and O. Hassager. Extensional viscosity for polymer melts measured in the filament stretching rheometer, Journal of Rheology, J. Rheol. 47, 429-441 (2003a).

[13] G. Marrucci and G. Ianniruberto, Interchain pressure effect in extensional flows of entangled polymer melts, Macromolecules 37, 3934-3942 (2004).

[14] M. H. Wagner, S. Kheirandish, and O. Hassager, Quantitative prediction of transient and steadystate elongational viscosity of nearly monodisperse polystyrene melts, J. Rheol. 49, 1317-1327 (2005).

[15] J. D. Schieber, D. M. Nair, and T. Kitkrailard, Comprehensive comparisons with nonlinear flow data of a consistently unconstrained Brownian slip-link model, J. Rheol. 51, 1111-1141 (2007).

[16] S. Dhole, A. Leygue, C. Bailly, and R. Keunings, A single segment differential tube model with interchain tube pressure effect, J. Non-Newtonian Fluid Mech. 161, 10-18 (2009).

[17] D. Auhl, P. Chambon, T. C. B. McLeish, and D. J. Read, Elongational flow of blends of long and short polymers: effective stretch relaxation time, Phys. Rev. Lett., 103, 136001 (2009).

[18] J. Read, K. Jagannathan, S. K. Sukumaran, and D. Auhl, A full-chain constitutive model for bidisperse blends of linear polymers, J. Rheol. 56, 823-873 (2012).

[19] M. L. Sentmanat, Miniature universal testing platform: from extensional melt rheology to solidstate deformation behavior, Rheol. Acta 43, 657-669 (2004).

[20] Y. Wang and S.-Q. Wang, From elastic deformation to terminal flow of a monodisperse entangled melt in uniaxial extension, J. Rheol. 52, 1275-1290 (2008). 
[21] Y. Wang, S. Cheng, and S.-Q. Wang, Basic characteristics of uniaxial extension rheology: Comparing monodisperse and bidisperse polymer melts, J. Rheol. 55, 1247-1270 (2011).

[22] M. H. Wagner and V. H. Rolón-Garrido, The interchain pressure effect in shear rheology, Rheol. Acta, 49, 459-471 (2010).

[23] J. K. Nielsen, H. K. Rasmussen, and O. Hassager, Stress relaxation of narrow molar mass distribution polystyrene following uni-axial extension, J. Rheol. 52, 885-899 (2008).

[24] J. K. Nielsen and H. K. Rasmussen, Reversed extension flow, J. Non-Newtonian Fluid Mech. 155, 15-19 (2008).

[25] Rasmussen, H. K., P. Laille, and K. Yu, Large amplitude oscillatory elongation flow , Rheol. Acta 47, 97-103 (2008).

[26] M. Andreev, R. N. Khaliullin, R. J. A. Steenbakkers, and J. D. Schieber, Approximations of the discrete slip-link model and their effect on nonlinear rheology predictions, J. Rheol. 57, 535-557 (2013).

[27] J. Park, D. W. Mead, M. M. Denn, Stochastic simulation of entangled polymeric liquids in fast flows: Microstructure modification, J. Rheol. 56, 1057-1081 (2012).

[28] K. K. Kabanemi, Kalonji K. and J.-F. Hetu, Dynamics of monodisperse linear entangled polymer melts in extensional flow: The effect of excluded-volume interactions, Polymer 50, 5865-5870 (2009).

[29] T. Yaoita, T. Isaki, Y. Masubuchi, H. Watanabe, G. Ianniruberto, G. Marrucci, Primitive Chain Network Simulation of Elongational Flows of Entangled Linear Chains: Stretch/Orientationinduced Reduction of Monomeric Friction, Macromolecules 45, 2773-2782 (2012).

[30] Baumgaertel, M., A. Schausberger, and H. H. Winter, The relaxation of polymers with linear flexible chains of uniform length, Rheol. Acta 29, 400-408 (1990).

[31] S. M. Fielding, Criterion for extensional necking instability in polymeric fluids, Phys. Rev. Lett. 107, 258301 (2011).

[32] J. K. Nielsen, , O. Hassager, H. K. Rasmussen, and G. H. McKinley, Observing the chain stretch transition in a highly entangled polyisoprene melt using transient extensional rheometry, J. Rheol. 53, 1327-1346 (2009).

[33] O. Hassager, M. I. Kolte, M. Renardy, Failure and nonfailure of fluid filaments in extension, J. Non-Newtonian Fluid Mech., 76, 137-151 (1998).

[34] K. Yu, J. M. R. Marin, H. K. Rasmussen, and O. Hassager, 3D modeling of dual wind-up extensional rheometers, J. Non-Newtonian Fluid Mech., 165, 14-23 (2010).

[35] H. K. Rasmussen and O. Hassager, The role of surface tension on the elastic decohesion of polymeric filaments, J. Rheol. 45, 527-537 (2001).

[36] H. K. Rasmussen, Lagrangian viscoelastic flow computations using the Rivlin-Sawyers constitutive model, J. Non-Newtonian Fluid Mech. 92, 227-243 (2000).

[37] H. K. Rasmussen, Lagrangian viscoelastic flow computations using a generalized molecular stress function model, J. Non-Newtonian Fluid Mech. 106, 107-120 (2002). 
[38] P. K. Currie,, Constitutive equations for polymer melts predicted by the Doi-Edwards and Curtiss-Bird kinetic theory models, J. Non-Newtonian Fluid Mech. 11, 53-68 (1982).

[39] K. Yu, H. K. Rasmussen, J. M. R. Marin, and O. Hassager, The dynamics of cylindrical samples in dual wind-up extensional rheometers, J. Rheol. 55, 571-580 (2011).

[40] A. Lyhne, H. K. Rasmussen, and O. Hassager, Simulation of elastic rupture in extension of entangled monodisperse polymer melts , Phys. Rev. Lett. 102, 138301 (2009).

[41] S. T. Milner and T. C. B. McLeish, Reptation and contour-length fluctuations in melts of linear polymers, Phys. Rev. Lett. 81, 725-728 (1998).

[42] Vinogradov, G. V., A. Y. Malkin, Y. G. Yanovskii, E. K. Borisenkova, B. V. Yarlykov, and G. V. Berenzhnaya, "Viscoelastic properties and flow of narrow distribution polybutadienes and polyisoprenes," J. Polymer Sci. 10 1061-1084 (1972).

[43] D. Auhl, J. Ramirez, A. E. Likhtman, P. Chambon, and C. Fernyhough, Linear and nonlinear shear flow behavior of monodisperse polyisoprene melts with a large range of molecular weights, J. Rheol. 52, 801-836 (2008).

[44] A. Bach, H. K. Rasmussen b, P.-Y. Longin, and O. Hassager, Growth of non-axisymmetric disturbances of the free surface in the filament stretching rheometer: experiments and simulation, J. Non-Newtonian Fluid Mech. 108, 163-186 (2002).

Table 1: Polymer melt parameters. The parameters $\left(\lambda_{\max }\right.$ and $\left.G_{N}^{0}\right)$ in equation 3 for the $240 \mathrm{~kg} / \mathrm{mole}$ SBR melt are obtained by fitting the data from Wang et al. [21]. The linear dynamics for the 575 $\mathrm{kg} /$ mole PI [42], shifted to $25^{\circ} \mathrm{C}$, agrees with the data for a $483 \mathrm{~kg} /$ mole PI [43], at $23^{\circ} \mathrm{C}$. The corresponding parameters in equation 3 for this PI are from Rasmussen et al. [44]

\begin{tabular}{l|lllll}
\hline Polymer & SBR & PS & PS & SBR & PI \\
$M_{w}[\mathrm{~kg} / \mathrm{mole}]$ & $95[2]$ & $390[11]$ & $465[3]$ & $240[21]$ & $575[42]$ \\
$M_{w} / M_{e}[]$ & $24[2]$ & $29[11]$ & $35[3]$ & $98[21]$ & $119[43]$ \\
Temp. $\left[{ }^{\circ} \mathrm{C}\right]$ & 23 & 150 & 150 & 25 & 25 \\
$\lambda_{\max }[\mathrm{s}]$ & $57.95[40]$ & $477[11]$ & $680[3]$ & 85 & $75.6[1,44]$ \\
$G_{N}^{0}[\mathrm{kPa}]$ & $652[40]$ & $270[11]$ & & 870 & $470[44]$ \\
$n_{e}[]$ & $0.261[40]$ & $0.16[11]$ & & $0.261[40]$ & $0.25[43]$ \\
$\tau_{R}[\mathrm{~s}]$ & $1.34[6]$ & $11[14]$ & & 0.48 & \\
\hline
\end{tabular}




\section{$6 \quad$ Figure Captions}

Figure 1: The solid lines are the dynamic development of the boundary contours in the finite element modeling (using the parameters for the $95 \mathrm{~kg} /$ mole SBR melt in table 1 and a $\dot{\epsilon}=0.1 \mathrm{~s}^{-1}$ ) where $L_{0} / R=12.7$. The dashed lines are symmetry lines. An initial perturbation of the cylindrical sample of $H / R=0.05$ and a width of $W / R=0.2$ is used in this finite element computation.

Figure 2: The smallest radius, $r(r(0)=R-H, z=0) / R$, as as function of the Hencky strain, $\epsilon$, obtained from the finite element computation in figure 1.

Figure 3: The strain of break $\epsilon_{c}$ as a function of the non-dimensional elongational rate $\dot{\epsilon} \cdot \lambda_{\max }$. The solid and dashed lines are calculated break for a cylindrical sample in a dual-wind up system with $L_{0} / R=12.7$, using the parameters in table 1 for the $95 \mathrm{~kg} /$ mole SBR melt (solid lines) and the 240 $\mathrm{kg} /$ mole SBR (dashed line). Initial perturbations of the cylindrical sample of depth $H / R=0.05$, $H / R=0.015$ and $H / R=0.005$ (from the bottom to the top solid curve) and a fixed width of $W / R=0.2$ are used. The dashed curve is at a $H / R=0.05$. The measured break of the $95 \mathrm{~kg} / \mathrm{mole}$ SBR melt from Wang and Wang [20] (•) and the $465 \mathrm{~kg} /$ mole PS from Luap et al. [3] (O).

Figure 4: The extension visocity, $\bar{\eta}^{+}=\left(\sigma_{11}-\sigma_{33}\right) / \dot{\epsilon}$, as a function of the time, $t$. The bullets $(\bullet)$, interconnected with dotted lines as a guide to the eye, are measurements for the $240 \mathrm{~kg} / \mathrm{mole}$ SBR melt from figure 2 and 12 in Wang et al. [21]. The extension rates $\dot{\epsilon}$ are $15 s^{-1}, 10 s^{-1}, 6 s^{-1}, 3 s^{-1}$, $1.0 s^{-1}, 0.3 s^{-1}$ and $0.1 s^{-1}$, from the left to the right curve. The solid lines are the corresponding theoretical prediction from the equations (1) and (2). The dashed line is the linear viscoelastic envelope. The used parameters are in table 1.

Figure 5: The strain of break $\epsilon_{c}$ as a function of the non-dimensional elongational rate $\dot{\epsilon} \cdot \lambda_{\max }$. The solid lines are calculated break for a cylindrical sample of length $L_{0} / R=12$, extended with fixtures moving in the axial direction, using the parameters for the $240 \mathrm{~kg} / \mathrm{mole}$ SBR melt in table 1. Initial perturbations of the cylindrical sample of depth $H / R=0.05, H / R=0.015$ and $H / R=0.005$ (from the bottom to the top curve) and a fixed width of $W / R=0.2$ are used. The measured break of the PI from Vinogradov et al. [1] (•) and the $240 \mathrm{~kg} /$ mole SBR melt from Wang et al. [21] (O). The dashed line is the same as in figure 3 . 


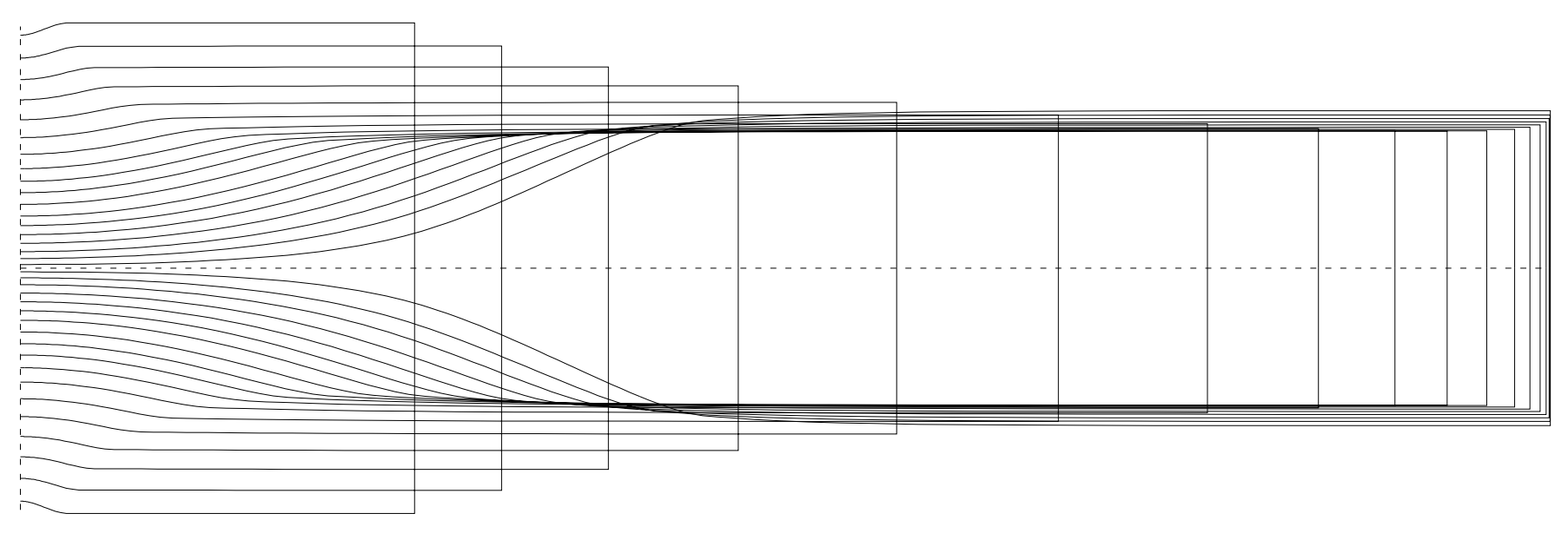

Figure 1: 


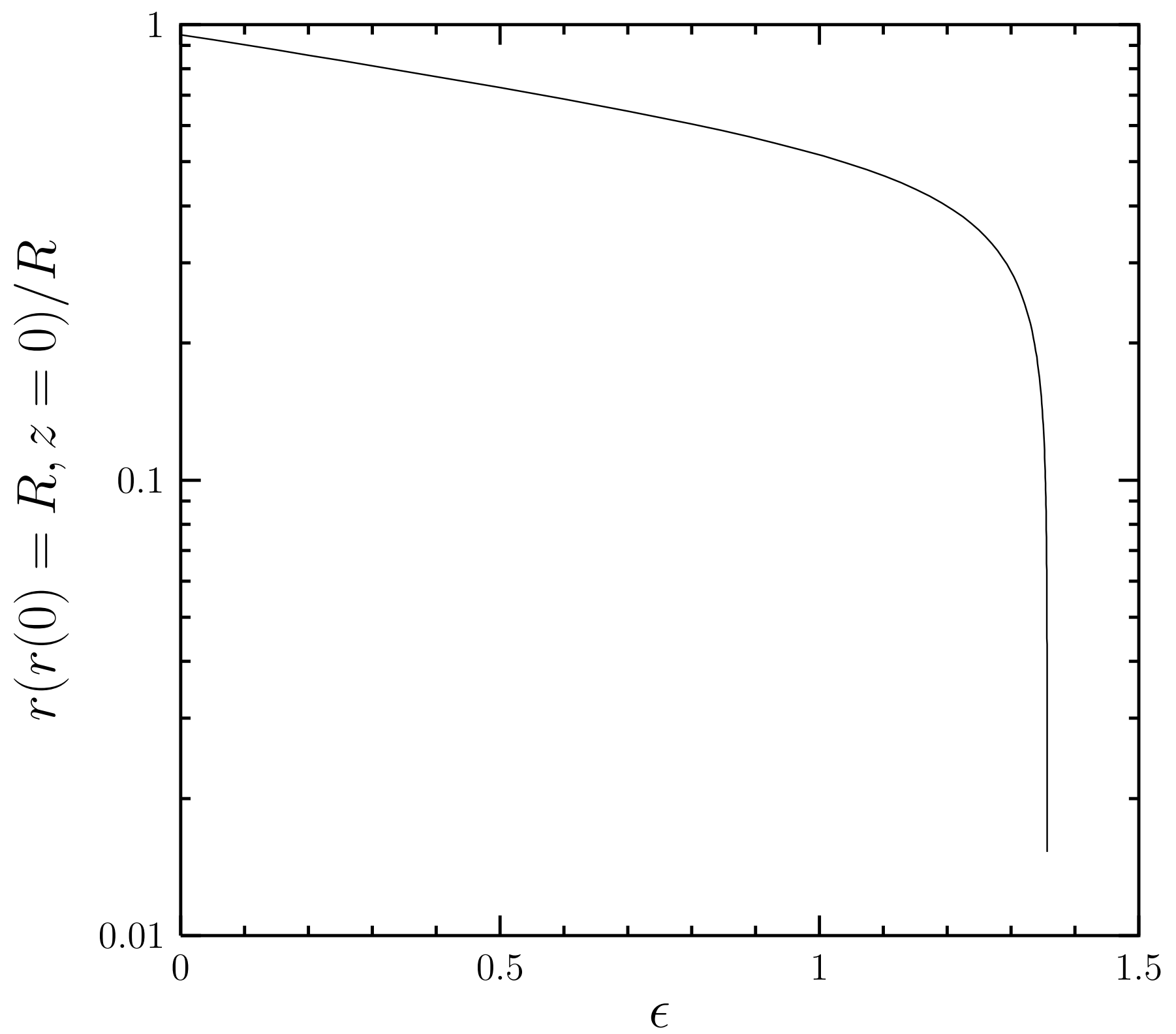

Figure 2: 


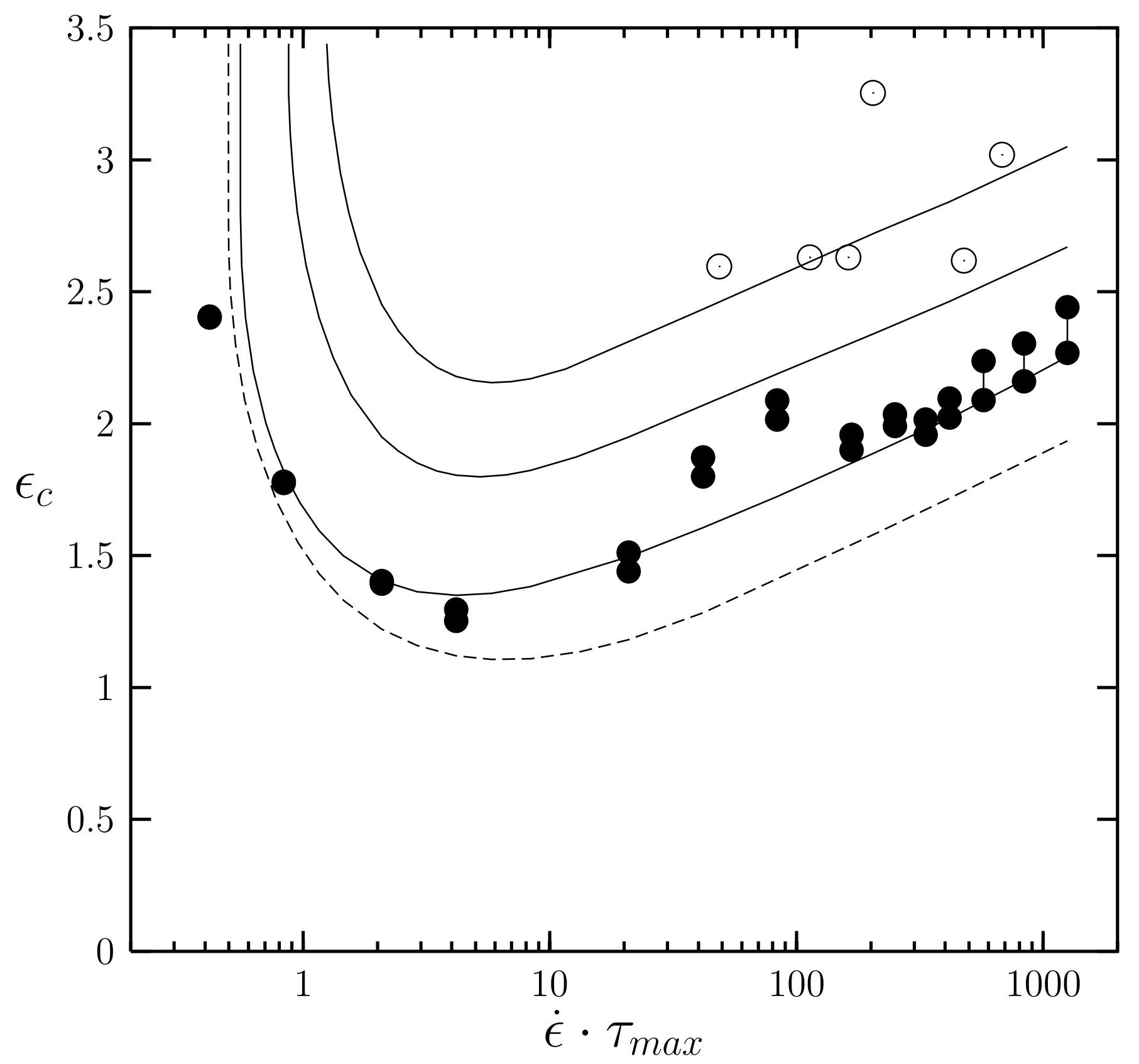

Figure 3: 


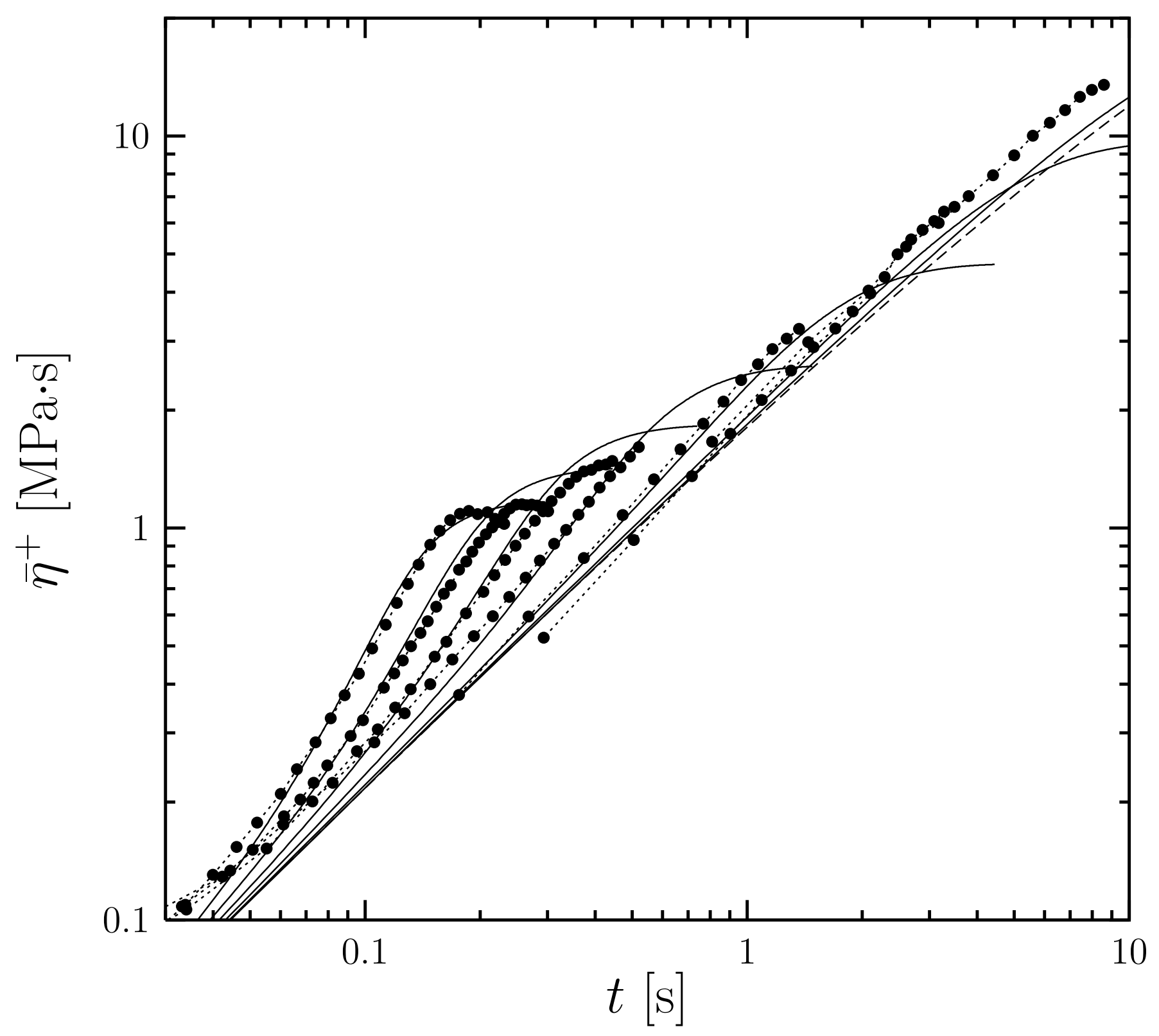

Figure 4: 


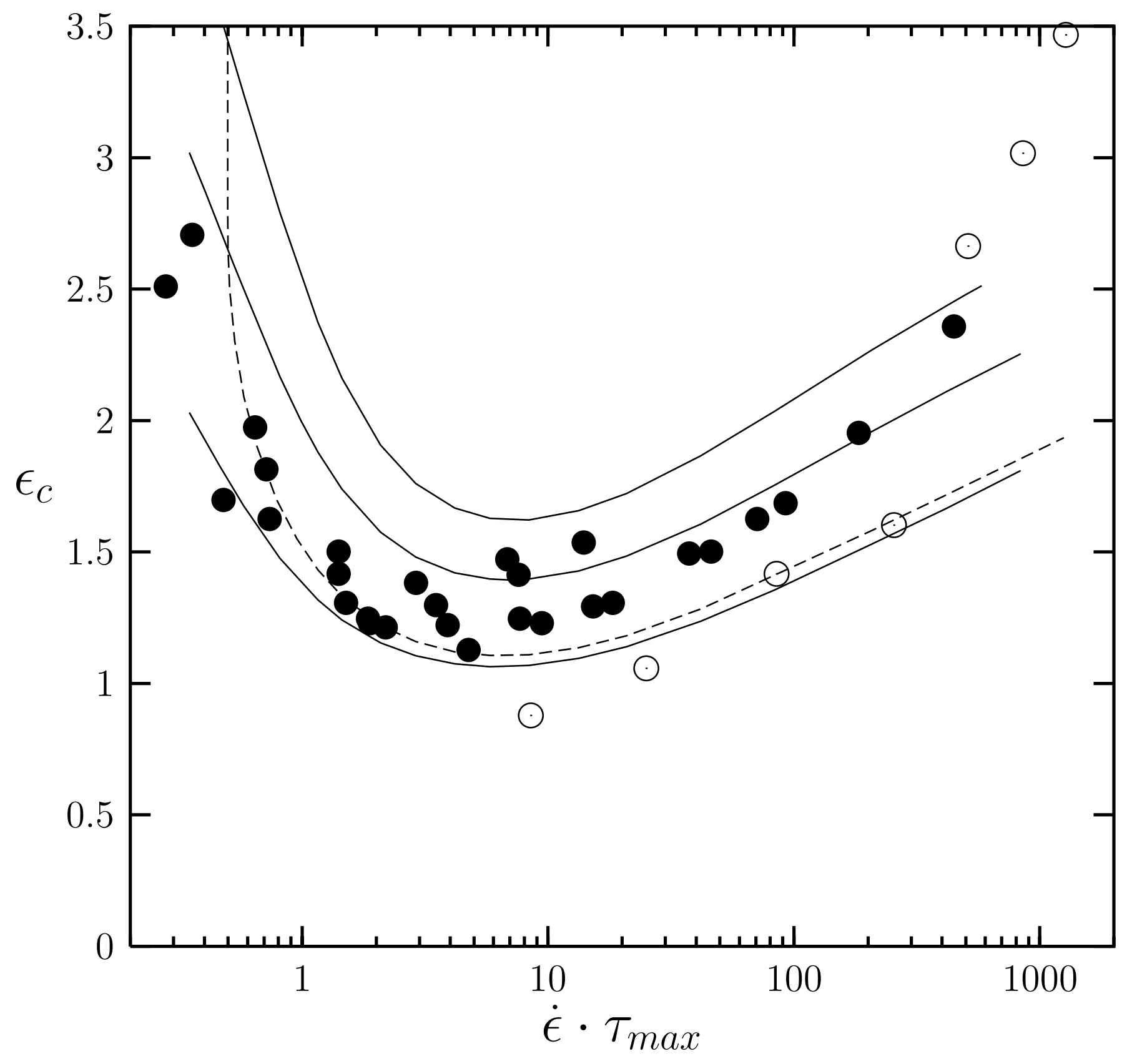

Figure 5: 